\title{
Preditores da intenção de permanecer em distanciamento social
}

\author{
Gabriel Horn Iwaya ${ }^{1}$ \\ Janaína Gularte Cardoso 23 \\ João Henriques de Sousa Júnior 2 \\ Andrea Valéria Steil 14 \\ 1 Universidade Federal de Santa Catarina / Programa de Pós-Graduação em Psicologia, Florianópolis / SC - Brasil \\ 2 Universidade Federal de Santa Catarina / Programa de Pós-Graduação em Administração, Florianópolis / SC - Brasil \\ 3 Universidade Federal da Fronteira Sul / Curso de Administração, Chapecó / SC - Brasil \\ ${ }^{4}$ Universidade Federal de Santa Catarina / Programa de Pós-Graduação em Engenharia e Gestão do Conhecimento, \\ Florianópolis / SC - Brasil
}

A presente pesquisa teve por objetivo desenvolver uma Escala de Intenção de Permanecer em Distanciamento Social (EIPDS) com base na Teoria do Comportamento Planejado (TCP). O modelo teórico estabelece que as variáveis "atitudes", "normas subjetivas" e "controle comportamental percebido" são preditoras da intenção de permanecer em distanciamento social (IPDS). O delineamento metodológico foi o de levantamento online com amostragem não probabilística. A pesquisa contou com 786 participantes brasileiros, majoritariamente residentes da região Sul do país. As variáveis da TCP explicaram $85 \%$ da variação da IPDS: normas subjetivas $(\beta=0,69)$, atitudes $(\beta=0,24)$ e controle comportamental percebido $(\beta=0,07)$. Os resultados evidenciam quais crenças subjacentes exercem influência sobre a formação da IPDS e podem servir às organizações públicas e privadas no processo de desenvolvimento de estratégias de promoção e engajamento da população na adoção do comportamento de "permanecer em distanciamento social" no Brasil. O trabalho apresenta as implicações práticas, as limitações da pesquisa e as possibilidades de estudos futuros sobre a IPDS.

Palavras-chave: COVID-19; intenção de permanecer em distanciamento social; distanciamento social.

\section{Predictores de la intención de permanecer en distanciamiento social}

Esta investigación tuvo como objetivo desarrollar una escala de intención de permanecer en distanciamiento social (EIPDS), con base en la teoría del comportamiento planificado (TCP). El modelo establece que las variables actitudes, normas subjetivas y control conductual percibido son predictoras de la intención de permanecer en distanciamiento social (IPDS). El diseño metodológico fue una encuesta online con muestreo no probabilístico. La encuesta contó con 786 participantes brasileños, en su mayoría residentes de la región sur del país. Las variables TCP explicaron el $85 \%$ de la variación de la IPDS: normas subjetivas $(\beta=0,69)$, actitudes $(\beta=0,24)$ y control conductual percibido $(\beta=0,07)$. Los resultados proporcionan evidencia sobre cuáles son las creencias subyacentes que influyen en la formación de la IPDS. Estas evidencias son relevantes para las organizaciones públicas y privadas en el proceso de desarrollo de estrategias de promoción, que generan el compromiso de la población de adoptar el comportamiento de "permanecer en distanciamiento social" en Brasil. En las discusiones, se presentan las implicaciones prácticas, señalando las limitaciones de la investigación y las posibilidades para futuros estudios sobre IPDS.

Palabras clave: COVID-19; intención de permanecer en distanciamiento social; distanciamiento social.

\section{Predictors of the intention to maintain social distancing}

This research aimed to develop an Intention to Maintain Social Distancing Scale (IMSDS) based on the Theory of Planned Behavior (TPB). The model establishes that the variables attitudes, subjective norms and perceived behavioral control are predictors of the intention to maintain social distancing (IMSD). The methodological design was an online survey with non-probabilistic sampling. The survey included 786 Brazilian participants, mostly residents of the southern region of the country. The TBP variables explained $85 \%$ of the IMSD variation: subjective norms $(\beta=0.69)$, attitudes $(\beta=0.24)$ and perceived behavioral control $(\beta=0.06)$. These results provide evidence of what are the underlying beliefs that influence the IMSD. This evidence is relevant to public and private organizations in the process of developing strategies to promote and engage the population in adopting the behavior of "maintaining social distancing" in Brazil. In the discussions, the practical implications are presented, pointing out the limitations of the research and the possibilities for future studies on IMSD.

Keywords: COVID-19; intention to maintain social distancing; social distancing. 


\section{INTRODUÇÃO}

A pandemia causada pelo novo coronavírus é considerada a mais grave ameaça à saúde pública mundial desde a primeira pandemia do vírus influenza H1N1, em 1918. Na ausência de uma vacina para o novo coronavírus, que levaria à contenção da atual doença, diversos países adotaram medidas de intervenção não farmacológicas com o objetivo de mitigar a transmissão do vírus, reduzindo os níveis de contato na população (Barros-Delben et al., 2020; Chaves \& Bellei, 2020; Lana et al., 2020). Entre as intervenções adotadas, a medida de distanciamento social está sendo amplamente utilizada, associada a ações governamentais de fechamento de comércios prescindíveis, escolas, universidades, etc.

Conforme a definição proposta pelo Imperial College, o distanciamento social ocorre quando "[...] todas as famílias reduzem o contato fora de casa, escola ou local de trabalho em 75\%" (Ferguson et al., 2020, p. 6). Entretanto, a realização efetiva do distanciamento social está associada a mudança de padrões comportamentais individuais, o que significa que cada pessoa precisa compreender a importância dessa medida para ser persuadida a adotar o comportamento de permanecer em distanciamento social. Estudos demonstram que o êxito desse processo de persuasão depende de uma comunicação efetiva das pessoas em posição de liderança em organizações públicas e privadas com a população (Allcott et al., 2020; Ferguson et al., 2020).

De acordo com a Teoria do Comportamento Planejado (TCP), comunicações persuasivas devem considerar o efeito que determinadas crenças estabelecidas pelas pessoas exercem sobre a formação da intenção de desempenhar um comportamento específico. A intenção é um cálculo subjetivo que engloba a decisão de uma pessoa para agir e a percepção do esforço que esta pessoa está disposta a realizar para executar um determinado comportamento (Abraham \& Sheeran, 2003). A intenção é o último estágio deliberativo antecessor à realização de um comportamento, sendo considerada o seu mais importante antecedente cognitivo (Ajzen, 1985, 1991, 2011).

Com base nos pressupostos da TCP, a presente pesquisa teve por objetivo desenvolver uma Escala de Intenção de Permanecer em Distanciamento Social (EIPDS). Embora a intenção seja um fenômeno universal (Ajzen, 2011), sua compreensão teórica é fortemente embasada em pesquisas realizadas na América do Norte e na Europa (Steinmetz, Knappstein, Ajzen, Schmidt, \& Kabst, 2016). Uma vez que o contexto influencia as crenças preditoras das intenções (Azjen, 2015), estudos devem ser realizados para a compreensão da dinâmica do desenvolvimento de intenções em diferentes contextos, culturas e momentos históricos (Leeuw, Valois, Ajzen, \& Schmidt, 2015; Steil, Floriani, Zilli, \& Rubio, 2019).

O desenvolvimento de uma escala, com a descrição de suas principais propriedades psicométricas, como validade e precisão, é uma etapa fundamental à mensuração e previsão de comportamentos (Draugalis, Coons, \& Plaza, 2008). Esse procedimento é um passo importante para assegurar que a escala realmente mensure os construtos pretendidos de uma forma reproduzível e confiável (Cohen, Swerdlik, \& Sturman, 2014). Dessa forma, a EIPDS desenvolvida neste trabalho pode ser utilizada como instrumento de mensuração da intenção das pessoas em aderir à prática do distanciamento social no Brasil.

Constataram-se, com o desenvolvimento da escala, quais crenças exercem maior efeito como preditoras da intenção de permanecer em distanciamento social; tais informações servem às organizações públicas na elaboração de estratégias de comunicação persuasivas voltadas à disseminação da prática do distanciamento social. A EIPDS desenvolvida neste trabalho também pode ser utilizada para avaliar amostras populacionais específicas, em contextos heterogêneos, considerando determinados recortes populacionais que se queira avaliar (ex. comunidades carentes, uma determinada cidade, universitários, etc.). 
De acordo com a TCP, todo o comportamento deliberado e consciente é determinado pela formação de uma intenção (Ajzen, 1985). Durante a formação da intenção, o sujeito elabora uma síntese das possíveis consequências e do esforço necessário para se comportar de determinado modo, baseado em crenças subjacentes estabelecidas ao longo de sua vida. Ao final deste processo psicológico, a intenção é formada e se torna o antecedente último do comportamento em si. O processo de formação da intenção, baseado em um conjunto de crenças e sua relação com o comportamento, constitui os objetos centrais da TCP (Ajzen, 1991, Fishbein \& Ajzen, 2010).

A TCP estabelece que a intenção de uma pessoa realizar um comportamento é influenciada por três conjuntos de crenças pessoais: atitudes, normas subjetivas e controle comportamental percebido. Hagger, Hamilton, Ajzen, Bosnjak, e Schmidt (2019) apontam que a eficácia da TCP já foi constatada por mais de duas mil pesquisas e trinta meta-análises. Uma vez demonstrada a eficácia do modelo teórico na explicação e predição de uma ampla gama de comportamentos em várias populações e contextos, foi utilizada a TCP no presente trabalho para explicar quais são os fatores antecedentes do comportamento de permanecer em distanciamento social.

De acordo com o modelo teórico adotado, a permanência em distanciamento social resulta de um processo deliberativo de tomada de decisão, determinado pela formação de uma intenção. Esta, por sua vez, é influenciada pelas atitudes relacionadas ao distanciamento social, pelas normas subjetivas e pelo controle comportamental percebido (Ajzen, 1985, 1991; Fishbein \& Ajzen, 2010).

As atitudes representam um conjunto de crenças comportamentais expressas na associação de atributos a um objeto. Para cada crença comportamental também é conferida uma avaliação subjetiva sobre a veracidade dessa associação. Considera-se que as pessoas possuam crenças relacionadas à medida de distanciamento social, incluindo a percepção das vantagens associadas ao comportamento (segurança, bem-estar, etc.). Quanto maior o grau de concordância (veracidade) com tais crenças, maior será a intenção de uma pessoa aderir à medida de distanciamento social (Ajzen, 1991; Fishbein \& Ajzen, 2010; Hagger et al., 2019). Assim, estabelece-se hipoteticamente que:

H1. As atitudes com relação ao distanciamento social influenciam a intenção de permanecer em distanciamento social.

As normas subjetivas referem-se às crenças normativas relacionadas à percepção da pressão social exercida sobre uma pessoa pelos seus pares. Tais crenças consideram a expectativa subjetiva de que as pessoas consideradas de referência para um indivíduo (ex. pais, família, amigos, cônjuge, colegas de trabalho, etc.) aprovem ou desaprovem determinado comportamento. A percepção da pressão social está atrelada também à motivação da pessoa para ceder à pressão (Ajzen, 1991; Fishbein \& Ajzen, 2010; Hagger et al., 2019). Considera-se que pessoas estejam em contato com outras que, por sua vez, também possuem crenças próprias a respeito da medida de distanciamento social. Quanto maior for a percepção, pelo indivíduo, de que pessoas importantes para ele acreditam que ele deva adotar a medida de distanciamento social, maior será a sua intenção de manter o comportamento. Assim, estabelece-se que:

H2. As normas subjetivas influenciam a intenção de permanecer em distanciamento social.

O controle comportamental percebido é representado por um conjunto de crenças relacionadas à percepção de fatores de controle (facilitadores ou impeditivos) associados ao desempenho do comportamento (Ajzen, 1991). Segundo Ajzen e Cote (2008), são considerados fatores de controle: habilidades requeridas, disponibilidade ou falta de tempo, dinheiro ou outros recursos, etc. Cada crença de controle contribui na formação do controle comportamental percebido, conformando 
um senso de autoeficácia individual (Fishbein \& Ajzen, 2010; Hagger et al., 2019). Quanto maior a percepção de controle sobre a permanência em distanciamento social, maior será a intenção de adotar tal comportamento. Desse modo, estabelece-se que:

H3. O controle comportamental percebido influencia a intenção de permanecer em distanciamento social.

\section{MÉTODO}

Esta é uma pesquisa quantitativa de levantamento online, com corte transversal e abordagem amostral por conveniência (Shaughnessy, Zechmeister, \& Zechmeister, 2012). A coleta de dados baseou-se em questionário divulgado em redes sociais (Facebook, Instagram e Whatsapp) no período de 31/03/2020 até 06/04/2020, com uma amostra majoritariamente constituída por moradores da região Sul do Brasil.

Foram estabelecidos dois critérios de participação: ter idade superior a 18 anos e ser do grupo de pessoas que podem escolher permanecer em distanciamento social. No início do questionário apresentou-se a definição de distanciamento social (Ferguson et al., 2020) e solicitou-se às pessoas que não pudessem escolher por permanecer em distanciamento social (ex. profissionais da área da saúde, de supermercados, farmácias, postos de combustíveis, etc.) que não respondessem à pesquisa. Para acessar ao questionário, os participantes precisavam responder afirmativamente à seguinte questão: você tem idade superior a 18 anos e pertence ao grupo de pessoas que pode escolher permanecer em distanciamento social ( $\operatorname{sim} /$ não)?

O questionário era composto por questões sociodemográficas (sexo biológico, idade, escolaridade, estado civil, ocupação, renda, estado e cidade) relacionadas aos construtos da TCP: atitudes (seis itens), normas subjetivas (seis itens), controle comportamental percebido (seis itens) e intenção de permanecer em distanciamento social (seis itens). Os itens foram desenvolvidos segundo as recomendações de Francis et al. (2004). A pesquisa adotou métodos de mensuração de intenção generalizada e medidas diretas, fazendo uso de escala do tipo Likert de sete pontos (discordo totalmente/concordo totalmente). Os itens utilizados para a mensuração das variáveis encontram-se no Quadro1.

\section{QUADRO 1 ITENS UTILIZADOS PARA A MENSURAÇÃO DAS VARIÁVEIS DA TCP}

$\begin{array}{cl}\begin{array}{c}\text { Itens utilizados para a mensuração das variáveis da Teoria do Comportamento Planejado (TCP) } \\ \text { Dimensão } \\ \text { teórica }\end{array} & \text { Itens } \\ & \text { ATT1 - Eu acho que permanecer em distanciamento social pelos próximos dias é uma escolha sensata. } \\ \text { ATT2 - Permanecer em distanciamento social pelos próximos dias faria eu me sentir bem. } \\ \text { ATT3 - Eu acho que permanecer em distanciamento social pelos próximos dias é uma escolha correta. } \\ \text { ATT4 - Permanecer em distanciamento social pelos próximos dias faria eu me sentir seguro(a). } \\ \text { ATT5 - Eu acho que permanecer em distanciamento social pelos próximos dias é uma escolha inteligente. } \\ \text { ATT6 - Permanecer em distanciamento social pelos próximos dias faria eu me sentir tranquilo(a). }\end{array}$




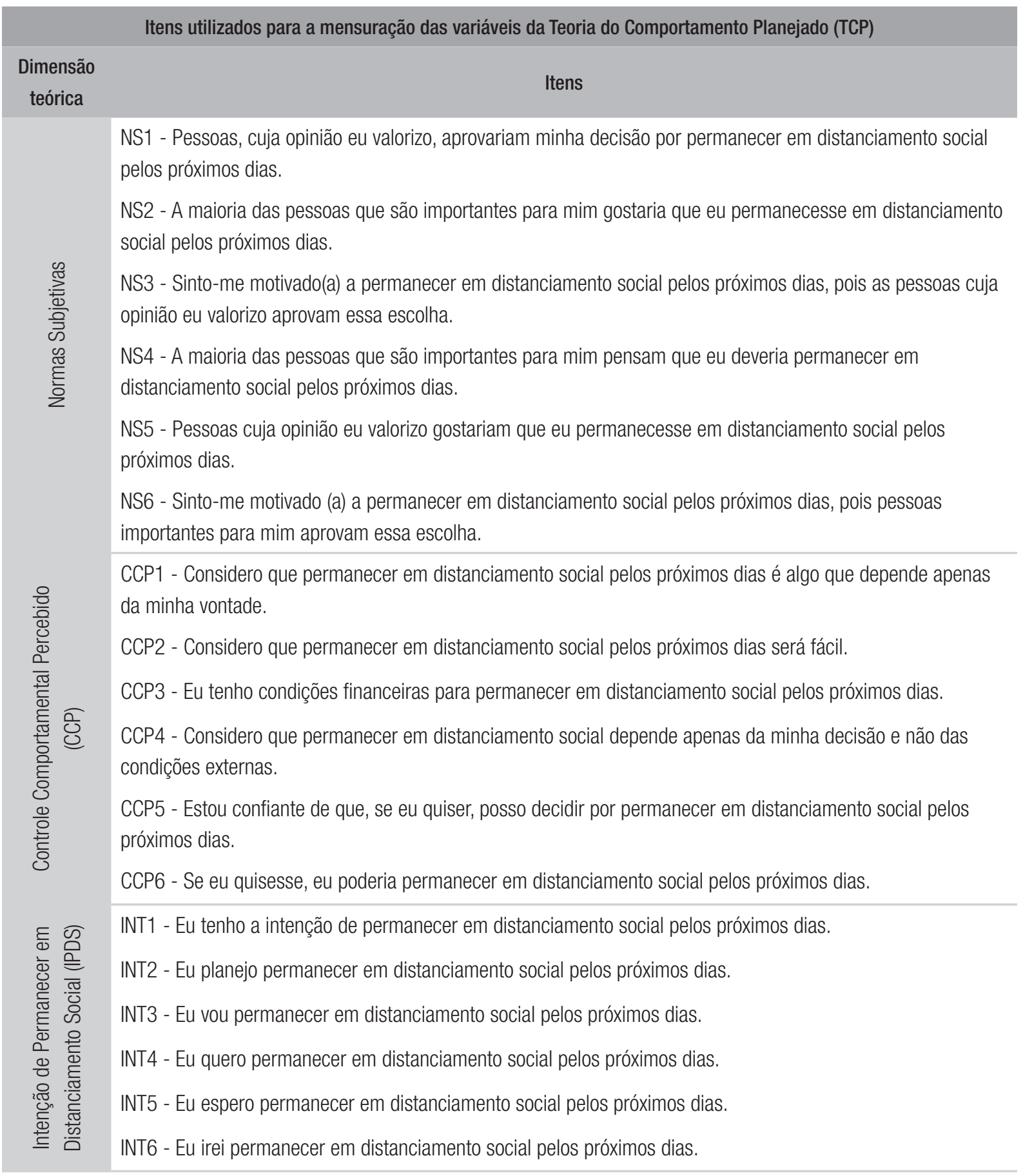

Fonte: Dados Primários (2020).

Para a análise da estrutura interna da EIPDS, utilizou-se a análise fatorial exploratória (AFE), método de fatoração pelo eixo principal e rotação oblíqua promax. A fatorabilidade dos dados foi avaliada triplamente: por meio da inspeção visual da matriz de correlação, do índice de Kaiser-MeyerOlkin $(\mathrm{KMO}>0,80)$ e do valor do teste de esfericidade de Bartlett $(\mathrm{p}<0,05)$. A extração de fatores foi feita por critérios a priori, com número fixo de quatro fatores (Damásio, 2012; Hair, Black, Babin, Anderson, \& Tatham, 2009). 
A solução fatorial obtida foi avaliada com base nos índices de comunalidade $\left(h^{2}>0,40\right)$ e cargas fatoriais $(>0,50)$. Variáveis com índices inaceitáveis e/ou cargas fatoriais cruzadas foram eliminadas. As reespecificações da análise foram feitas até que fosse encontrada uma estrutura fatorial adequada. A análise de confiabilidade foi avaliada pelo índice do alfa de Cronbach $(\alpha>0,70)$ (Hair et al., 2009).

Conforme recomendam Ajzen (1991) e Francis et al. (2004), a mensuração do efeito das atitudes, normas subjetivas e CCP foi feita por meio de regressão linear múltipla hierárquica. Foram consideradas as seguintes suposições: retirada de outliers acima de três desvios padrão; ausência de multicolinearidade $(r>0,80)$, observando os valores de tolerância $(>0,10)$ e VIF $(<10)$; homocedasticidade, observando a distribuição dos pontos do gráfico de dispersão; independência dos resíduos, observando os valores do teste de Durbin-Watson (aceitável entre 1,50 e 2,50); erros normalmente distribuídos, observando o histograma e gráfico P-P normal de regressão de resíduos padronizados; e relação linear entre as variáveis, observando o gráfico de dispersão (Hair et al., 2009).

\section{RESULTADOS}

A pesquisa contou com 786 participantes, de 17 estados do Brasil, distribuídos entre 109 municípios. A maioria dos participantes reside na região Sul do país (88\%), com a seguinte distribuição: Santa Catarina (80\%), Rio Grande do Sul (6\%) e Paraná (2\%). O perfil sociodemográfico dos participantes pode ser observado na Tabela 1.

\section{TABELA 1 PERFIL DOS RESPONDENTES}

\begin{tabular}{lcc}
\multicolumn{1}{c}{ Variáveis Sociodemográficas } & Frequências & Percentagem \\
\hline Sexo biológico & 486 & \\
Feminino & 300 & 61,80 \\
Masculino & & 38,20 \\
Faixa etária & 378 & \\
$18-27$ anos & 211 & 48,09 \\
$28-37$ anos & 104 & 26,84 \\
$38-47$ anos & 59 & 13,23 \\
$48-57$ anos & 29 & 7,51 \\
$58-67$ anos & 5 & 3,69 \\
$68-77$ anos & & 0,64 \\
\hline
\end{tabular}


RAP | Preditores da intenção de permanecer em distanciamento social

\begin{tabular}{|c|c|c|}
\hline \multicolumn{3}{|c|}{ PERFIL SOCIODEMOGRÁFICO DOS PARTICIPANTES DA PESQUISA (N=786) } \\
\hline Variáveis Sociodemográficas & Frequências & Percentagem \\
\hline \multicolumn{3}{|l|}{ Nível de escolaridade } \\
\hline Ensino fundamental & 1 & 0,13 \\
\hline Ensino médio & 322 & 40,97 \\
\hline Ensino superior & 213 & 27,10 \\
\hline Pós-graduação (especialização) & 103 & 13,10 \\
\hline Mestrado & 79 & 10,05 \\
\hline Doutorado & 68 & 8,65 \\
\hline \multicolumn{3}{|l|}{ Estado civil } \\
\hline Solteiro(a) & 491 & 62,50 \\
\hline Casado(a)/União estável & 249 & 31,70 \\
\hline Divorciado(a)/Separado(a)/Viúvo(a) & 41 & 5,80 \\
\hline \multicolumn{3}{|l|}{ Ocupação } \\
\hline Sem vínculo empregatício & 236 & 34,99 \\
\hline Funcionário de organização privada & 174 & 22,14 \\
\hline Servidor público & 170 & 21,63 \\
\hline Profissional autônomo e/ou liberal & 112 & 14,25 \\
\hline Empresário/Empregador de organização privada & 24 & 3,05 \\
\hline Estagiário(a) de organização pública e/ou privada & 12 & 1,53 \\
\hline Aposentado(a)/Pensionista & 9 & 1,15 \\
\hline Pesquisador(a)/Bolsista & 7 & 0,89 \\
\hline Empregado(a) de sociedade de economia mista & 2 & 0,25 \\
\hline Agricultor(a) & 1 & 0,15 \\
\hline \multicolumn{3}{|l|}{ Renda familiar } \\
\hline Até 3 salários mínimos & 306 & 38,90 \\
\hline Entre 3 e 6 salários mínimos & 209 & 26,60 \\
\hline Entre 6 e 9 salários mínimos & 107 & 13,60 \\
\hline Entre 9 e 12 salários mínimos & 72 & 9,20 \\
\hline Entre 12 e 15 salários mínimos & 44 & 5,60 \\
\hline Acima de 15 salários mínimos & 48 & 6,10 \\
\hline
\end{tabular}

Fonte: Dados Primários (2020).

O perfil dos participantes caracterizou-se pela prevalência de pessoas do sexo biológico feminino (61,80\%), de idade entre 18 e 27 anos (48,09\%), com ensino médio completo (40,97\%), solteiras $(62,50 \%)$, com renda familiar de até 3 salários mínimos (38,90\%) e sem vínculo empregatício (35\%). 


\subsection{Análise fatorial exploratória}

A hipótese nula de normalidade dos itens da EIPDS foi rejeitada pelo teste de KolmogorovSmirnov ( $\mathrm{p}<0,000$ ), por isso utilizou-se o método de fatoração pelo eixo principal [principal axis factoring] (Damásio, 2012). Os itens apresentaram correlações entre si com intensidades que não ultrapassaram 0,90 , sugerindo ausência de alta multicolinearidade ou sobreposição de itens (Pasquali, 2005). O tamanho da amostra foi considerado aceitável (superior a trinta casos por item) (Hair et al., 2009).

A análise inicial de fatorabilidade dos dados foi feita por meio da inspeção visual da matriz de correlação, da medida KMO e do teste de esfericidade de Bartlett, sendo considerada aceitável. A inspeção da matriz de correlação apresentou número substancial de correlações maiores que 0,30. A medida KMO foi considerada excelente $(0,96)$. O teste de Bartlett confirmou a adequação da amostragem para aplicação da análise fatorial $\left[\chi^{2}(276)=21.043,56, p<0,00\right]$.

A exibição dos coeficientes na solução fatorial foi ordenada pelo tamanho de suas cargas, ocultando os coeficientes menores que 0,30. Em sequência, foram avaliadas as cargas e a comunalidade das variáveis. Segundo Hair et al. (2009), em uma perspectiva conservadora, podem-se eliminar os itens com comunalidade menores que 0,50 .

A flexibilização do valor mínimo de comunalidade é uma prática comumente empregada por estudos exploratórios (Castillo, Macrini, Cheniaux, \& Landeira-Fernandez, 2010; Pereira et al., 2019). Nesta pesquisa, foram eliminados os itens com valor de comunalidade menor que 0,40 . Na análise fatorial inicial, os itens CCP2 $(0,35)$ e CCP3 $(0,31)$ apresentaram baixa comunalidade $\left(\mathrm{h}^{2}>0,40\right)$ e o item CCP3 também não apresentou carga fatorial em quaisquer dimensões. Dessa forma, estes itens foram excluídos, sendo refeita a análise fatorial.

Os itens NS3 e NS6 apresentaram cargas cruzadas nas dimensões atitudes e normas subjetivas. Os itens INT4 e INT5 apresentaram cargas fatoriais cruzadas nas dimensões atitudes e intenção de permanecer em distanciamento social. Após a exclusão destes quatro itens, os itens ATT1, ATT3, ATT5, CCP6 começaram a apresentar cargas cruzadas com os itens da dimensão da intenção de permanecer em distanciamento social. Todos os dez itens precisaram ser retirados (ATT1, ATT3, ATT5, NS3, NS6, CCP2, CCP3, CCP6, INT4 e INT5), sendo excluídos um de cada vez.

Para além das observações estatísticas que explicam a exclusão dos itens supracitados, outros pontos podem ser observados. Os itens excluídos ATT1, ATT3 e ATT5 representavam crenças cognitivas com relação à medida de distanciamento social, em que se considerava a adoção da medida como algo sensato, correto e inteligente. Os itens remanescentes que consolidaram a dimensão das atitudes estavam relacionados a crenças afetivas (sentir-se bem, tranquilo, seguro). No escopo da TCP, atitudes são compostas por componentes afetivos e cognitivos das crenças vinculadas ao comportamento. Reconhece-se que eles são dificilmente separados na prática e nem sempre permanecem em exata correspondência entre si (Schleicher, Watt, \& Greguras, 2004). Por exemplo, quando pensamos no comportamento de manutenção do distanciamento social, temos sentimentos sobre o que pensamos. Da mesma maneira, quando sentimos algo com relação à manutenção do distanciamento social, pensamos nestes sentimentos. Na amostra estudada, verificou-se que as crenças de conteúdo afetivo foram mais consistentes como medidas da atitude relacionadas ao distanciamento social. Isso pode representar o quanto a adoção da medida de distanciamento social está mobilizando aspectos afetivos na vida das pessoas neste momento de pandemia. 
Os itens excluídos NS3 e NS6 representavam crenças normativas relacionadas à motivação para atender a pressão social percebida, sendo a motivação uma faceta subjacente das normas subjetivas. $\mathrm{Na}$ EIPDS havia itens que se referiam especificamente à percepção da pressão social (NS1, NS2, NS4, NS5) e itens que se referiam à motivação individual para atender a pressão social. A exclusão dos itens relacionados à motivação sugere que tais medidas não se correlacionam efetivamente com a dimensão das normas subjetivas relativamente ao comportamento de permanecer em distanciamento social. A origem latina da palavra "motivação" significa "mover" e assim ela é amplamente difundida no senso comum. Contudo, o significado científico de "motivação" é o estudo da ação e sua relação com crenças, valores, metas e objetivos (Eccles \& Wigfield, 2002). Embora ela tenha este significado científico, a percepção popular do termo é mais ampla e é possível que o significado pretendido não tenha sido compreendido pelos respondentes.

Os itens excluídos CCP2, CCP3 e CCP6 representavam, respectivamente, crenças de controle relacionadas à dificuldade percebida (fácil/difícil), ao controle financeiro (ter dinheiro para) e à autoeficácia (se eu quisesse). Os itens remanescentes (CCP1, CCP4, CCP5) estão relacionados com a percepção de autoeficácia quanto à adoção da medida de distanciamento social. Vale destacar que os índices obtidos na análise fatorial dos itens remanescentes da dimensão de controle comportamental percebido foram os que apresentaram menores comunalidades, o que pode estar associado ao fato de a medida de distanciamento social ser uma recomendação inédita para a população. Provavelmente os participantes estão vivenciando pela primeira vez a necessidade da adoção desse tipo de comportamento e não possuem experiências que possam servir de parâmetro para tecer avaliações sobre fatores impeditivos e/ou facilitadores relacionados ao desempenho de tal comportamento.

Os itens INT4 e INT5 apresentaram cargas cruzadas nas dimensões atitudes e intenção de permanecer em distanciamento social. De certo modo, teoricamente era esperada a existência de algum grau de correlação entre as dimensões fatoriais atitudes e intenção de permanecer em distanciamento social, bem como é possível que as crenças comportamentais relacionadas à dimensão das atitudes correlacionem-se com as crenças que dizem respeito à intenção. Pressupondo-se a existência de colinearidade entre as dimensões, utilizou-se o método de rotação oblíqua promax na análise fatorial explanatória (Hair et al., 2009). Após a exclusão dos itens INT4 e INT5, os itens remanescentes consolidaram uma dimensão fatorial própria com maior consistência interna.

Após a exclusão dos itens, um de cada vez, uma nova inspeção era feita. O processo foi realizado até que os problemas de carregamentos cruzados fossem resolvidos e as cargas fatoriais apresentassem valores aceitáveis $(>0,50)$. A análise de confiabilidade dos conjuntos de itens, avaliada pelo alfa de Cronbach, também demonstrou valores aceitáveis $(\alpha>0,70)$. A solução fatorial final obtida pode ser observada na Tabela 2. 


\section{TABELA 2 ANÁLISE FATORIAL EXPLORATÓRIA}

\section{ANÁLISE FATORIAL DE EXTRAÇÃO PELO EIXO PRINCIPAL, ROTAÇÃO PROMAX, COMUNALIDADES ( $\left.h^{2}\right)$ E ALFA DE CRONBACH DA ESCALA DE INTENÇÃO DE PERMANECER EM DISTANCIAMENTO SOCIAL (EIPDS).}

\begin{tabular}{|c|c|c|c|c|c|}
\hline Item & Fator 1 & Fator 2 & Fator 3 & Fator 4 & $h^{2}$ \\
\hline $\begin{array}{l}\text { NS4 - A maioria das pessoas que são importantes para mim pensam } \\
\text { que eu deveria permanecer em distanciamento social pelos próximos } \\
\text { dias. }\end{array}$ & 0,96 & & & & 0,83 \\
\hline $\begin{array}{l}\text { NS5 - Pessoas cuja opinião eu valorizo gostariam que eu } \\
\text { permanecesse em distanciamento social pelos próximos dias. }\end{array}$ & 0,92 & & & & 0,83 \\
\hline $\begin{array}{l}\text { NS2 - A maioria das pessoas que são importantes para mim gostaria } \\
\text { que eu permanecesse em distanciamento social pelos próximos dias. }\end{array}$ & 0,89 & & & & 0,76 \\
\hline $\begin{array}{l}\text { NS1 - Pessoas cuja opinião eu valorizo aprovariam minha decisão } \\
\text { por permanecer em distanciamento social pelos próximos dias }\end{array}$ & 0,54 & & & & 0,60 \\
\hline $\begin{array}{l}\text { INT3 - Eu vou permanecer em distanciamento social pelos próximos } \\
\text { dias. }\end{array}$ & & 1,05 & & & 0,87 \\
\hline $\begin{array}{l}\text { INT6 - Eu irei permanecer em distanciamento social pelos próximos } \\
\text { dias. }\end{array}$ & & 0,94 & & & 0,85 \\
\hline $\begin{array}{l}\text { INT2 - Eu planejo permanecer em distanciamento social pelos } \\
\text { próximos dias. }\end{array}$ & & 0,80 & & & 0,84 \\
\hline $\begin{array}{l}\text { INT1 - Eu tenho a intenção de permanecer em distanciamento social } \\
\text { pelos próximos dias. }\end{array}$ & & 0,63 & & & 0,83 \\
\hline $\begin{array}{l}\text { ATT6 - Permanecer em distanciamento social pelos próximos dias } \\
\text { faria eu me sentir tranquilo(a). }\end{array}$ & & & 0,98 & & 0,78 \\
\hline $\begin{array}{l}\text { ATT2 - Permanecer em distanciamento social pelos próximos dias } \\
\text { faria eu me sentir bem. }\end{array}$ & & & 0,75 & & 0,62 \\
\hline $\begin{array}{l}\text { ATT4 - Permanecer em distanciamento social pelos próximos dias } \\
\text { faria eu me sentir seguro(a). }\end{array}$ & & & 0,61 & & 0,77 \\
\hline $\begin{array}{l}\text { CCP4 - Considero que permanecer em distanciamento social } \\
\text { depende apenas da minha decisão e não das condições externas. }\end{array}$ & & & & 0,88 & 0,51 \\
\hline $\begin{array}{l}\text { CCP1 - Considero que permanecer em distanciamento social pelos } \\
\text { próximos dias é algo que depende apenas da minha vontade. }\end{array}$ & & & & 0,76 & 0,44 \\
\hline $\begin{array}{l}\text { CCP5 - Estou confiante de que, se eu quiser, posso decidir por } \\
\text { permanecer em distanciamento social pelos próximos dias. }\end{array}$ & & & & 0,53 & 0,43 \\
\hline Autovalor & 8,14 & 1,88 & 0,91 & 0,74 & \\
\hline Variância Explicada (\%) & 58,18 & 13,45 & 6,47 & 5,26 & \\
\hline Número de Itens & 4 & 4 & 3 & 3 & \\
\hline Alfa de Cronbach & 0,93 & 0,96 & 0,90 & 0,78 & \\
\hline
\end{tabular}

Fonte: Dados Primários (2020). 
A solução fatorial final apresentou medida KMO excelente $(0,92)$, e o teste de esfericidade de Bartlett evidenciou a rejeição da hipótese nula, o que confirma a adequação da amostragem para aplicação da análise fatorial $\left[\chi^{2}(91)=10.816,67, p<0,00\right]$. A solução fatorial indicou uma estrutura com quatro dimensões, com variância explicativa acumulada de $81,36 \%$.

A EIPDS obtida é composta por quatorze itens, distribuídos em quatro dimensões. O Fator 1 (normas subjetivas) é composto por quatro itens, os quais respondem por $56,18 \%$ da variabilidade dos dados $(\alpha=0,93)$. O Fator 2 (intenção de permanecer em distanciamento social) é composto por quatro itens, os quais respondem por $13,45 \%$ da variabilidade dos dados $(\alpha=0,96)$. O Fator 3 (atitudes) é composto por três itens, os quais respondem por $6,47 \%$ da variabilidade dos dados $(\alpha=0,90)$. O Fator 4 (controle comportamental percebido) é composto por três itens, os quais respondem por $5,26 \%$ da variabilidade dos dados $(\alpha=0,78)$.

Tomando-se por base o conjunto de itens que constituiu cada fator, foram calculadas as variáveis: atitudes, normas subjetivas, controle comportamental percebido e intenção de permanecer em distanciamento social. $\mathrm{O}$ valor atribuído às variáveis deve corresponder, respectivamente, à média aritmética dos itens em cada dimensão fatorial (Hair et al., 2009). Os valores mínimos, máximos, as médias e o desvio padrão das variáveis podem ser observados na Tabela 3.

\section{TABELA 3 ÍNDICES DE DISPERSÃO DAS VARIÁVEIS}

\begin{tabular}{lcccc}
\multicolumn{4}{c}{ ÍNDICES DE DISPERSÃ0 DAS VARIÁVEIS } & \\
\multicolumn{1}{c}{ Variáveis } & Mínimo & Máximo & Média & Desvio Padrão \\
\hline 1. Atitudes & 1,00 & 7,00 & 5,75 & 1,64 \\
2. Normas Subjetivas & 1,00 & 7,00 & 6,20 & 1,31 \\
3. Controle Comportamental Percebido & 1,00 & 7,00 & 3,97 & 1,75 \\
4. IPDS & 1,00 & 7,00 & 6,16 & 1,52
\end{tabular}

Fonte: Dados Primários (2020).

A média da intenção de permanecer em distanciamento social $(6,16)$ sugere que a amostra concentra participantes que se consideram altamente inclinados à adoção da medida. A média das normas subjetivas $(6,20)$ indica que os participantes sentem-se altamente pressionados a permanecer em distanciamento social. A média das atitudes $(5,75)$ demonstra que os participantes também se apresentam favoráveis à medida de distanciamento social, enquanto a média do controle comportamental percebido $(3,97)$ sugere que a percepção de controle dos participantes distribui-se de forma mais heterogênea. Os resultados indicam que os participantes apresentam maior probabilidade de adotar a medida de distanciamento social, possivelmente devido à amostra ser não probabilística, o que não garante que cada elemento da população brasileira tenha igual chance de ser incluído na amostra (Shaughnessy et al., 2012). É possível que as pessoas mais inclinadas a permanecer em distanciamento social e/ou que já estavam adotando a medida tenham se sentido mais motivadas e/ou estivessem mais disponíveis para responder a pesquisa. 


\subsection{Análise de regressão linear múltipla hierárquica}

A técnica de regressão múltipla hierárquica foi utilizada para verificar se as variáveis atitudes, normas subjetivas e controle comportamental percebido preveem a intenção de permanecer em distanciamento social. A inclusão das três variáveis independentes foi feita uma a uma, gerando três modelos. Um quarto modelo ainda foi testado para verificar possíveis efeitos das variáveis sociodemográficas. A análise de outliers identificou sessenta casos de valores residuais discrepantes, os quais foram excluídos da análise. A amostra utilizada foi de 726 participantes. Todas as suposições para a realização da técnica de regressão foram atendidas. O resumo dos modelos testados pode ser observado na Tabela 4.

\section{TABELA 4 RESUMO DOS MODELOS TESTADOS}

\begin{tabular}{|c|c|c|c|c|c|c|c|c|c|}
\hline \multicolumn{10}{|c|}{ RESUMO DOS MODELOS TESTADOS } \\
\hline \multirow[b]{2}{*}{ Modelo } & \multirow[b]{2}{*}{ R } & \multirow[b]{2}{*}{$\mathrm{R}^{2}$} & \multirow[b]{2}{*}{$\mathrm{R}^{2}$ ajustado } & \multirow{2}{*}{$\begin{array}{c}\text { Erro } \\
\text { padrão }\end{array}$} & \multicolumn{5}{|c|}{ Estatísticas de mudança } \\
\hline & & & & & $\begin{array}{c}\text { Mudança } \\
\text { de } \mathrm{R}^{2}\end{array}$ & Mudança F & df1 & df2 & $\mathrm{p}$-valor \\
\hline 1 & 0,81 & 0,65 & 0,65 & 0,79 & 0,65 & $1.342,47$ & 1,00 & 724 & 0,00 \\
\hline 2 & 0,92 & 0,84 & 0,84 & 0,53 & 0,19 & 856,66 & 1,00 & 723 & 0,00 \\
\hline 3 & 0,92 & 0,84 & 0,84 & 0,53 & 0,00 & 16,52 & 1,00 & 722 & 0,00 \\
\hline 4 & 0,92 & 0,85 & 0,85 & 0,52 & 0,01 & 4,14 & 6,00 & 716 & 0,00 \\
\hline
\end{tabular}

Notas:

Modelo 1: (Constante), Atitudes.

Modelo 2: (Constante), Atitudes, Normas Subjetivas.

Modelo 3: (Constante), Atitudes, Normas Subjetivas, Controle Comportamental Percebido.

Modelo 4: (Constante), Atitudes, Normas Subjetivas, Controle Comportamental Percebido, Faixa Etária, Renda, Escolaridade, Ocupação, Sexo Biológico, Estado Civil.

Variável Dependente: Intenção de Permanecer em Distanciamento Social.

Fonte: Dados Primários (2020).

A análise resultou em um modelo estatisticamente significativo $[\mathrm{F}(10,715)=401,51, \mathrm{p}<0,00$, $\mathrm{R}^{2}=0,85, \mathrm{R}^{2}$ ajustado $\left.=0,85\right]$. As atitudes $(\beta=0,24, \mathrm{t}=10,17, \mathrm{p}<0,00)$, normas subjetivas $(\beta=0,69, \mathrm{t}=28,82, \mathrm{p}<0,00)$ e o controle comportamental percebido $(\beta=0,07, \mathrm{t}=4,29, \mathrm{p}<0,00)$ são preditores da intenção de permanecer em distanciamento social. Todas as hipóteses (H1, H2 e H3) lançadas por esta pesquisa foram aceitas. As variáveis da TCP explicaram $85 \%$ da variação da intenção de permanecer em distanciamento social. Hierarquicamente as atitudes contribuíram com 65\% do total de variância explicada pelo modelo, as normas subjetivas contribuem com $19 \%$, o controle comportamental percebido contribuiu com $0,4 \%$ e as variáveis sociodemográficas contribuíram com $0,5 \%$.

O acréscimo das variáveis sociodemográficas revelou que as variáveis faixa etária $(\beta=-0,07$, $\mathrm{t}=-3,79, \mathrm{p}<0,00)$, renda $(\beta=0,04, \mathrm{t}=2,22, \mathrm{p}<0,03)$ e escolaridade $(\beta=0,04, \mathrm{t}=1,98, \mathrm{p}<0,05)$ são preditoras da intenção de permanecer em distanciamento social. Entretanto, as variáveis ocupação $(\beta=-0,03, \mathrm{t}=-1,87, \mathrm{p}<0,06)$, sexo biológico $(\beta=-0,01, \mathrm{t}=-0,85, \mathrm{p}<0,40)$ e estado civil $(\beta=-0,01$, 
$\mathrm{t}=-0,46, \mathrm{p}<0,65)$ não apresentaram efeito, considerando nível de significância de $95 \%$. O efeito das variáveis sociodemográficas foi considerado inexpressivo $(\beta \leq 0,20)$. A interpretação dos coeficientes de regressão ( $\beta$ ) seguiu o critério de Cohen (1992): pequeno $(\beta \geq 0,20)$, médio $(\beta \geq 0,50)$ e grande $(\beta \geq 0,70)$. Os coeficientes não padronizados $(B)$, padronizados $(\beta)$ e o teste de significância dos modelos testados são apresentados na Tabela 5.

\section{TABELA 5 COEFICIENTES E TESTE DE SIGNIFICÂNCIA}

\begin{tabular}{|c|c|c|c|c|c|}
\hline \multicolumn{6}{|c|}{ COEFICIENTES NÃO PADRONIZADOS (B), PADRONIZADOS ( $\beta$ ) E TESTE DE SIGNIFICÂNCIA } \\
\hline Modelos & B & $\begin{array}{c}\text { Erro } \\
\text { padrão }\end{array}$ & $\beta$ & $\mathrm{T}$ & $\mathrm{p}$-valor \\
\hline \multicolumn{6}{|l|}{ Modelo 1} \\
\hline (Constante) & 2,33 & 0,11 & & 20,56 & 0,00 \\
\hline Atitudes & 0,68 & 0,02 & 0,81 & 36,64 & 0,00 \\
\hline \multicolumn{6}{|l|}{ Modelo 2} \\
\hline (Constante) & 0,37 & 0,10 & & 3,69 & 0,00 \\
\hline Atitudes & 0,21 & 0,02 & 0,25 & 10,18 & 0,00 \\
\hline Normas Subjetivas & 0,75 & 0,03 & 0,71 & 29,27 & 0,00 \\
\hline \multicolumn{6}{|l|}{ Modelo 3} \\
\hline (Constante) & 0,28 & 0,10 & & 2,75 & 0,01 \\
\hline Atitudes & 0,20 & 0,02 & 0,24 & 9,74 & 0,00 \\
\hline Normas Subjetivas & 0,74 & 0,03 & 0,70 & 29,23 & 0,00 \\
\hline Controle Comportamental Percebido & 0,05 & 0,01 & 0,06 & 4,06 & 0,00 \\
\hline \multicolumn{6}{|l|}{ Modelo 4} \\
\hline (Constante) & 0,34 & 0,12 & & 2,78 & 0,01 \\
\hline Atitudes & 0,21 & 0,02 & 0,24 & 10,17 & 0,00 \\
\hline Normas Subjetivas & 0,73 & 0,03 & 0,69 & 28,82 & 0,00 \\
\hline Controle Comportamental Percebido & 0,05 & 0,01 & 0,07 & 4,29 & 0,00 \\
\hline Faixa etária & $-0,08$ & 0,02 & $-0,07$ & $-3,79$ & 0,00 \\
\hline Renda & 0,04 & 0,02 & 0,04 & 2,22 & 0,03 \\
\hline Escolaridade & 0,04 & 0,02 & 0,04 & 1,98 & 0,05 \\
\hline Ocupação (1, com ocupação; 0, sem ocupação) & $-0,09$ & 0,05 & $-0,03$ & $-1,87$ & 0,06 \\
\hline Sexo Biológico (1, feminino; 0, masculino) & $-0,03$ & 0,04 & $-0,01$ & $-0,85$ & 0,40 \\
\hline Estado Civil (1, Solteiro(a); 0, outros) & $-0,02$ & 0,05 & $-0,01$ & $-0,46$ & 0,65 \\
\hline
\end{tabular}

Nota: $\mathrm{R}^{2}$ ajustado $=0,65$ para o modelo $1 ; \Delta \mathrm{R}^{2}=0,19$ para o modelo $2 ; \Delta \mathrm{R}^{2}=0,004$ para o modelo $3 ; \Delta \mathrm{R}^{2}=0,005$ para o modelo 4 . Fonte: Dados Primários (2020). 
As atitudes e as normas subjetivas apresentaram altas correlações $(r>0,50)$ com a intenção de permanecer em distanciamento social, enquanto o controle comportamental percebido apresentou média correlação $(0,30)$. As atitudes e as normas subjetivas também apresentam alta correlação entre si $(0,79)$, enquanto as correlações entre as atitudes e as normas subjetivas com o controle comportamental percebido foram baixas $(r<0,30)$. A interpretação dos coeficientes de correlações $(r)$ foi feita pelo critério de Cohen (1992): $r \geq 0,10$ (fraca), $r \geq 0,30$ (média) e $r \geq 0,50$ (alta). As correlações entre as variáveis podem ser observadas na Tabela 6.

\section{TABELA 6 MATRIZ DE CORRELAÇÃO DAS VARIÁVEIS}

\begin{tabular}{|c|c|c|c|c|}
\hline \multicolumn{5}{|c|}{ MATRIZ DE CORRELAÇÃO DAS VARIÁVEIS } \\
\hline Variáveis & 1 & 2 & 3 & 4 \\
\hline 1. Atitudes & 1,00 & & & \\
\hline 2. Normas Subjetivas & $0,79^{*}$ & 1,00 & & \\
\hline 3. Controle Comportamental Percebido & $0,27^{*}$ & $0,25^{\star}$ & 1,00 & \\
\hline 4. Intenção de Permanecer em Distanciamento Social & $0,80^{*}$ & $0,90^{*}$ & $0,30^{\star}$ & 1,00 \\
\hline
\end{tabular}

Em virtude das correlações encontradas, pode-se dizer que as atitudes e as normas subjetivas apresentam uma parcela de variância explicativa compartilhada da intenção de permanecer em distanciamento social, enquanto o controle comportamental percebido explica melhor uma parcela própria da variância explicativa da intenção, conforme a Tabela 5. No modelo 02 , com o acréscimo das normas subjetivas, as atitudes têm seu efeito reduzido. No modelo 03, a adição do controle comportamental percebido não interferiu no efeito das atitudes e das normas subjetivas.

\section{DISCUSSÃ0}

O objetivo desta pesquisa foi desenvolver uma EIPDS com base na TCP, amplamente utilizada em estudos nas áreas de Saúde e Ciências Humanas. A meta-análise de Armitage e Conner (2001) apresenta evidências sobre a eficácia da utilização da TCP em diferentes contextos, com uma explicação média de $39 \%$ da variação da intenção. O modelo aqui empregado explicou $85 \%$ da variação da IPDS, demonstrando que a TCP é eficaz para explicar a formação desta intenção. Mediante o peso relativo de cada preditor (atitudes, normas subjetivas e controle comportamental percebido) da IPDS, podese prever o comportamento de permanência em distanciamento social e, principalmente, planejar intervenções para este fim.

A variável normas subjetivas, relacionada à percepção da pressão social exercida sobre as pessoas pelos seus pares, foi o preditor que mais influenciou a formação da IPDS neste estudo. Ou seja, quanto mais uma pessoa percebe que pessoas importantes para ela pensam que ela deveria permanecer em distanciamento social, mais disposição ela apresenta para aquiescer à pressão social. A percepção das vantagens pessoais associadas à permanência em distanciamento social (crenças atitudinais 
positivas), como bem-estar e segurança, também influenciou a formação da IPDS. O acréscimo do preditor controle comportamental percebido melhorou o ajuste do modelo, embora seu efeito tenha sido próximo de zero $(\beta=0,06)$. O mesmo pode ser observado sobre o efeito da inclusão das variáveis sociodemográficas no modelo.

Os resultados da pesquisa são relevantes para organizações públicas e privadas envolvidas com o combate à COVID-19, pois evidenciam que as crenças de conteúdos normativos e atitudinais exercem alta e média influência, respectivamente, sobre a intenção de permanecer em distanciamento social. Sendo assim, elas são úteis para a elaboração de estratégias que promovam a permanência em distanciamento social. Tais resultados oferecem um arcabouço conceitual e reforçam as propostas de Van Bavel et al. (2020) sobre possíveis respostas que as ciências sociais podem oferecer à pandemia da COVID-19.

De acordo com Van Bavel et al. (2020), identificar figuras-chave credíveis (ex. líderes religiosos ou políticos) pode ser uma estratégia eficaz na disseminação de mensagens persuasivas em campanhas de saúde pública durante epidemias. Esses autores destacam que, durante a crise de Ebola na África Ocidental, o engajamento dos líderes religiosos em campanhas que defendiam boas práticas foi considerado o ponto de virada na resposta à epidemia.

Van Bavel et al. (2020) indicam também que os líderes devem reforçar seu apoio, livre de associações político-partidárias, às medidas relacionadas à COVID-19 - nos casos onde essa discordância ocorre -, pois esse endosso reduz a polarização política ao redor do tema e auxilia na promoção das medidas. Van Bavel et al. (2020) salientam que as mensagens devem procurar enfatizar: a) os benefícios aos destinatários (ex. autoproteção); b) o comportamento como uma medida de proteção social; c) apelos normativos (ex. "a esmagadora maioria das pessoas em sua comunidade acredita que todos devem ficar em casa"); d) o comportamento como algo esperado pelos grupos sociais.

Uma análise destas recomendações, com base na TCP, indica que líderes atuam como pessoas significativas capazes de fomentar a pressão social para o desempenho de um comportamento. Portanto eles são fomentadores de normas subjetivas que pressionam as pessoas a se comportarem de um modo específico. Como os resultados desta pesquisa mostraram, as normas subjetivas e as atitudes positivas relacionadas ao distanciamento social estão correlacionadas, por isso a importância desses líderes é dupla. Eles têm poder e legitimidade para disseminar informações favoráveis ao comportamento alvo. Ou seja, influenciam a formação das normas subjetivas e das atitudes, exatamente as duas variáveis com maior força na formação da intenção de permanecer em distanciamento social.

Diante dessa constatação, o cenário ideal seria o de um discurso uníssono entoado à população pelas figuras que exercem papel de liderança, sejam elas políticas, religiosas, artísticas, sociais, digitais, etc. Porém o cenário de polarização política existente no Brasil não só fortalece o nível de discordância entre os discursos apresentados, como também trata da questão da saúde pública como um embate de ideais partidários, desvencilhado das necessárias evidências que justifiquem suas ações.

Ajzenman, Cavalcanti e Da Mata (2020) revelam resultados alarmantes com relação ao impacto do discurso anti-isolamento propagado pelo presidente Bolsonaro sobre o comportamento das pessoas. Os pesquisadores combinaram dados eleitorais e dados geográficos de telefonia móvel de mais de 60 milhões de dispositivos em todo o país. As evidências encontradas pelos pesquisadores apontam que, depois de o presidente do Brasil descartar pública e enfaticamente os riscos associados à COVID-19 e desaconselhar o isolamento, os índices de distanciamento social de cidadãos em localidades pró-governo diminuíram significativamente. 
Levanta-se a hipótese de que a orientação político-partidária das pessoas exerça efeito sobre a intenção de permanecer em distanciamento social. Essa influência foi constatada pelo estudo de Allcott et al. (2020). De acordo com estes autores, o conteúdo das mensagens de líderes políticos e dos principais meios de comunicação sobre a gravidade da COVID-19 afeta substancialmente a forma como os cidadãos norte-americanos respondem à situação pandêmica. Os autores ainda destacam que as questões político-partidárias entre republicanos e democratas, nos Estados Unidos, não trazem resultados positivos para nenhum dos lados. A falta de consenso entre os discursos sobre o combate à pandemia gera conflitos e desinformação, atrapalha o combate da doença e, potencialmente, gera um custo econômico maior, ao contrário do que ocorreria se os dois lados políticos adotassem as mesmas crenças e o mesmo discurso de conscientização da população (Allcott et al., 2020).

No Brasil, o estudo feito por Farias e Pilati (2020) evidencia o efeito do partidarismo político sobre a intenção de violar a medida de distanciamento social. O levantamento online realizado pelos pesquisadores contou com a participação de 2.056 brasileiros (amostragem não probabilística). De acordo com Farias e Pilati (2020), pessoas que apoiam o presidente Bolsonaro apresentam maior intenção de violar as medidas de distanciamento social. Conforme os autores pontuam, no Brasil, Bolsonaro é visto como um político conservador de extrema direita que se posicionou veementemente contra a medida de distanciamento social, alegando que tal medida causaria impacto negativo à economia. Como consequência, pessoas que compartilham da sua identidade partidária tendem a apoiá-lo e questionar a adoção da medida. Neste contexto, Farias e Pilati (2020) concordam com Van Bavel et al. (2020) acerca da importância de se reduzir a polarização política em torno das ações de combate à pandemia.

O presente estudo investigou especificamente o efeito que os preditores originais da TCP (atitudes, normas subjetivas, controle comportamental percebido) exercem sobre a formação da intenção de permanecer em distanciamento social. Diversas pesquisas utilizam a TCP como um modelo de referência, propondo extensões do modelo com a adição de novos construtos, prática recomendada por Ajzen (1991), tendo como objetivo observar se a adição de novos construtos aumentaria sua capacidade preditiva. Entretanto, primeiramente, recomenda-se a testagem do modelo da TCP com suas variáveis originais, verificando-se se elas explicam, ou não, uma parcela significativa da variância da intenção.

Desse modo, as evidências apresentadas por Allcott et al. (2020) e Farias e Pilati (2020) sugerem que a adição de um construto voltado à orientação político-partidária, como possível preditor da adoção do distanciamento social, pode ser considerada por estudos futuros. Outras variáveis com potencial preditivo podem estar associadas à percepção de casos próximos e das consequências deletérias da doença, como conhecer alguém próximo: a) que tenha sido infectado pelo vírus; b) que ficou com sequelas respiratórias após contrair a doença; c) e/ou que veio a óbito. Estudos que investiguem a percepção de casos próximos e das consequências deletérias da doença no Brasil ainda não foram divulgados.

Os autores recomendam que estudos futuros que se utilizem da EIPDS apliquem-na integralmente, reintegrando, também, os itens que foram excluídos no processo de análise fatorial exploratória, verificando possíveis variações decorrentes da aplicação da escala integral em outras amostras e com pessoas que vivenciam diferentes circunstâncias e/ou contextos. Recomenda-se também um segundo estudo que aplique técnicas de modelagem de equações estruturais, verificando a robustez do instrumento e realizando análises do modelo de mensuração e do modelo estrutural. 
Estudos futuros também devem ponderar a viabilidade de: a) utilizar outros tipos de delineamento de pesquisa (experimentais e/ou longitudinais); b) incorporar uma medida do comportamento real; c) utilizar abordagens amostrais representativas e/ou estratificadas; d) verificar diferenças no padrão de respostas dos participantes quando a adoção da medida for estabelecida por tempo indeterminado. As últimas sugestões encontram-se intrinsecamente ligadas às limitações metodológicas apresentadas nesta seção.

À guisa de conclusão, esta pesquisa constitui um esforço inicial voltado à construção de uma EIPDS, ancorada nos pressupostos da TCP, o qual contribui para a compreensão da eficácia da EIPDS na mensuração dos construtos pretendidos de uma forma reproduzível e confiável. A escala desenvolvida apresentou propriedades psicométricas aceitáveis e pode ser utilizada como instrumento de mensuração da intenção das pessoas em aderir à prática do distanciamento social no Brasil.

Esta pesquisa fornece também informações relevantes sobre quais crenças exercem maior efeito como preditoras da intenção de permanecer em distanciamento social. Foi constatado que as normas subjetivas relacionadas com a percepção da pressão social percebida exercem forte influência sobre a intenção de permanecer em distanciamento social. As atitudes relacionadas com a percepção de vantagens (ex. bem-estar, segurança) também exerceram média influência sobre a intenção de permanecer em distanciamento social. Tais informações podem ser consideradas por organizações públicas e privadas para elaboração de estratégias de comunicação persuasivas voltadas à disseminação da prática do distanciamento social.

\subsection{Limitações}

Como todo estudo, este também apresenta limitações metodológicas. Fishbein e Ajzen (2010) estabelecem duas categorias principais de limitações relacionadas às pesquisas que utilizam a TCP como modelo preditor da intenção: a) limitações relacionadas à validade da avaliação dos construtos por meio de um delineamento de levantamento, b) limitações relacionadas ao uso de dados correlacionais para testagem de efeitos causais.

Críticas associadas ao delineamento de levantamento devem-se principalmente à utilização de medidas de autorrelato, que podem apresentar viés de consistência relacionado à desejabilidade social e resultar em correlações superestimadas entre os componentes teóricos. Sobre a limitação relacionada ao uso de dados correlacionais para testagem de efeitos causais, Fishbein e Ajzen (2010) comentam que dados correlacionais podem ser interpretados parcialmente como evidências de efeitos causais (preditivos), dadas as evidências robustas que suportam a TCP.

A amostragem não probabilística e a coleta de dados online também implicam limitações. A amostra utilizada nesta pesquisa não pode ser considerada representativa da população brasileira, por isso seus resultados não podem ser generalizados e sua interpretação deve ser cautelosa. A observação dos dados sociodemográficos evidencia que a amostra apresenta viés de seleção, com super-representação de um segmento da população (por exemplo, $48 \%$ de participantes na faixa etária de 18 até 27 anos, 58,90\% dos participantes com ensino superior ou acima, 88\% dos participantes residentes na região Sul do Brasil).

Outra limitação diz respeito à questão adotada para triagem dos participantes ("você tem idade superior a 18 anos e pertence ao grupo de pessoas que pode escolher permanecer em distanciamento social?"), cujo potencial para gerar viés de resposta leva o participante a refletir sobre sua possibilidade 
de aderir a um comportamento, ao passo que outras pessoas não têm tal possibilidade. Essa limitação poderia ter sido sanada com a apresentação da pergunta de triagem ao término do questionário.

Estudos têm demonstrado que a avaliação subjetiva de atributos associados a comportamentos pode variar em virtude da distância temporal percebida entre a formulação da intenção e o comportamento real (Ajzen, 1985). A avaliação de crenças sobre a adoção da medida de distanciamento social também deve considerar a percepção do tempo de permanência nessa situação. Este estudo avaliou a intenção de permanecer em distanciamento social estabelecendo que essa permanência seria "pelos próximos dias". Se a percepção do tempo de adoção da medida de distanciamento social fosse alterada (ex. "enquanto for requisitado pelos órgãos competentes"), certamente haveria mudanças no padrão de respostas dos participantes. 


\section{REFERÊNCIAS}

Abraham, C., \& Sheeran, P. (2003). Acting on intentions: the role of anticipated regret. British Journal of Social Psychology, 42(4), 495-511. Recuperado de http://doi.org/10.1348/014466603322595248

Ajzen, I. (1985). From intentions to actions: a theory of planned behavior. In J. Kuhl, \& J. Beckmann (Eds.), Action control (SSSP Springer Series in Social Psicology, pp. 11-39). Berlin, Germany: Heidelberg. Recuperado de https://link.springer.com/chapt er/10.1007/978-3-642-69746-3_2

Ajzen, I. (1991). The theory of planned behavior. Organizational behavior and human decision processes, 50(2), 179-211. Recuperado de http://doi. org/10.1016/0749-5978(91)90020-T

Ajzen, I., \& Cote, N. G. (2008). Attitudes and the prediction of behavior. In W. D. Crano, \& R. Prislin (Eds.), Attitudes and attitude change (pp. 289-311). New York, NY: Psychology Press.

Ajzen, I. (2011). The theory of planned behaviour: reactions and reflections. Psychology \& Health, 26(9), 1113-1127. Recuperado de http://doi.org/10.1080/0 8870446.2011 .613995

Ajzen, I. (2015). The theory of planned behaviour is alive and well, and not ready to retire: a commentary on Sniehotta, Pressau, and AraújoSoares. Health Psychology Review, 9(2), 131-137. Recuperado de http://doi.org/10.1080/17437199 .2014 .883474

Ajzenman, N., Cavalcanti, T., \& Da Mata, D. (2020, 23 de abril). More than words: leaders' speech and risky behavior during a pandemic. SSRN. Recuperado de http://dx.doi.org/10.2139/ssrn.3582908

Allcott, H., Boxell, L., Conway, J. C., Gentzkow, M., Thaler, M., \& Yang, D. Y. (2020, abril). Polarization and public health: Partisan differences in social distancing during the Coronavirus pandemic (Working Paper n. 26946). National Bureau of Economic Research. SSRN. Recuperado de https:// ssrn.com/abstract $=3574415$

Armitage, C. J., \& Conner, M. (2001). Efficacy of the theory of planned behaviour: a metaanalytic review. British journal of social psychology, 40(4), 471-499. Recuperado de https://doi. org/10.1348/014466601164939
Barros-Delben, P., Cruz, R. M., Trevisan, K.R. R., Gai, M. J. P., Carvalho, R. V. C., Carlotto, P. A. C. ... Malloy-Diniz, L. F. (2020, junho). Saúde mental em situações de emergência: COVID-19. Revista Debates in Psiquiatria, Ano 10(2), 18-28. Recuperado de https://d494f813-3c95-463a-898c-ea1519530871. filesusr.com/ugd/c37608_6bd285d2f02b40098a94c8 1c49d603b8.pdf

Castillo, C., Macrini, L., Cheniaux, E., \& LandeiraFernandez, J. (2010). Psychometric properties and latent structure of the Portuguese version of the Penn State Worry Questionnaire. The Spanish journal of psychology, 13(1), 431-443. Recuperado de https:// doi.org/10.1017/S113874160000398X

Chaves, T. S. S., \& Bellei, N. (2020). SARS-CoV-2, o novo Coronavírus: uma reflexão sobre a Saúde Única (One Health) e a importância da medicina de viagem na emergência de novos patógenos. Revista de Medicina, 99(1), 1-4. Recuperado de http://dx.doi. org/10.11606/issn.1679-9836.v99i1 pi-iv

Cohen, J. (1992). A power primer. Psychological Bulletin, 112(1), 155-159. Recuperado de https:// doi.org/10.1037/0033-2909.112.1.155

Cohen, R. J., Swerdlik, M. E., \& Sturman, E. D. (2014). Testagem e Avaliação Psicológica: introdução a teste e medidas. Porto Alegre, RS: Artmed.

Damásio, B. F. (2012). Uso da análise fatorial exploratória em psicologia. Avaliação Psicológica, 11(2), 213-228. Recuperado de https:// pepsic.bvsalud.org/pdf/avp/v11n2/v11n2a07.pdf

Draugalis, J. R., Coons, S. J., \& Plaza, C. M. (2008). Best practices for survey research reports: A synopsis for authors and reviewers. American Journal of Pharmaceutical Education, 72(1), 1-6. Recuperado de https://doi.org/10.5688/aj720111

Eccles, J. S. \& Wigfield, A. (2002). Motivational beliefs, values, and goals. Annual Review of Psychology, 53(1), 109-132. Recuperado de https:// doi.org/10.1146/annurev.psych.53.100901.135153

Farias, J. E. M., \& Pilati, R. (2020, 29 de abril). Violating social distancing amid COVID-19 pandemic: psychological factors to improve compliance. PsyArXiv Preprints. Recuperado de https://doi.org/10.31234/osf.io/apg9e

Ferguson, N., Laydon, D., Nedjati Gilani, G., Imai, N., Ainslie, K., Baguelin, M. ... Dighe, A. 
(2020, 16 de março). Report 9: Impact of nonpharmaceutical interventions (NPIs) to reduce COVID-19 mortality and healthcare demand. London, UK: Imperial College. Recuperado de https://doi.org/10.25561/77482

Fishbein, M., \& Ajzen, I. (2010). Predicting and changing behavior: the reasoned action approach. New York, NY: Psychology Press. Recuperado de https://doi.org/10.4324/9780203838020

Francis, J., Eccles, M. P., Johnston, M., Walker, A. E., Grimshaw, J. M., Foy, R. ... Bonetti, D. (2004). Constructing questionnaires based on the theory of planned behaviour: a manual for health services researchers. Newcastle upon Tyne, UK: Centre for Health Services Research, University of Newcastle upon Tyne. Recuperado de http://openaccess.city. ac.uk/1735/

Hagger, M. S., Hamilton, K., Ajzen, I., Bosnjak, M., \& Schmidt, P. (2019, novembro). Testing the replicability of the Theory of Planned Behavior: a large-scale multisample registered replication study. Trier, German: Leibniz Institut für Psychologische Information und Dokumentation [ZPID]. Recuperado de http:// dx.doi.org/10.23668/psycharchives.2652

Hair, J. F., Black, W. C., Babin, B. J., Anderson, R. E., \& Tatham, R. L. (2009). Análise multivariada de dados. Porto Alegre, RS: Bookman.

Lana, R. M., Coelho, F. C., Gomes, M. F. D. C., Cruz, O. G., Bastos, L. S., Villela, D. A. M., \& Codeço, C. T. (2020). Emergência do novo coronavirus (SARS-CoV-2) e o papel de uma vigilância nacional em saúde oportuna e efetiva. Cadernos de Saúde Pública, 36(3), 1-5. Recuperado de https://doi. org/10.1590/0102-311X00019620

Leeuw, A., Valois, P., Ajzen, I., \& Schmidt, P. (2015). Using the theory of planned behavior to identify key beliefs underlying pro-environmental behavior in high-school students: implications for educational interventions. Journal of Environmental Psychology, 42(1), 128-138. Recuperado de https:// doi.org/10.1016/j.jenvp.2015.03.005

Pasquali, L. (2005). Análise fatorial para pesquisadores. Brasília, DF: LabPAM.

Pereira, C. D. A., Maciel, S. C., Dias, C. C. V., Alexandre, T. M. D. O., Oliveira, M. X. D., \& Pimentel, C. E. (2019). Validação da Escala de Crenças sobre Abuso Sexual (ECAS) no contexto brasileiro. Psico-USF, 24(1), 145-158. Recuperado de https://doi.org/10.1590/1413-82712019240112

Schleicher D. J., Watt J. D., \& Greguras G. J. (2004). Reexamining the job satisfaction-performance relationship: the complexity of attitudes. Journal of applied psychology, 89(1), 165-77. Recuperado de https://doi.org/10.1037/0021-9010.89.1.165

Shaughnessy, J. J., Zechmeister, E. B., \& Zechmeister, J. S. (2012). Metodologia de pesquisa em psicologia. Porto Alegre, RS: AMGH.

Steil, A. V., Floriani, E. V., Zilli, M., \& Rubio, M. A. (2019). Intenção de sair da organização: definições, métodos e citações em publicações nacionais. Revista Psicologia: Organizações e Trabalho, 19(2), 580-587. Recuperado de https://doi.org/10.17652/ rpot/2019.2.14080

Steinmetz, H.; Knappstein, M.; Ajzen, I., Schmidt, P.; \& Kabst, R. (2016). How effective are behaviour change interventions based on the theory of planned behaviour? A three level meta-analysis. Zeitschrift für Psychologie, 224(3), 2016-233. Recuperado de https://doi.org/10.1027/2151-2604/a000255

Van Bavel, J. J., Baicker, K, Boggio, P., Capraro, V., Cichocka, A., Crockett, M., ... Willer, R. (2020, 24 de março). Using social and behavioural science to support COVID-19 pandemic response. PsyArXiv Preprints. Recuperado de https://doi.org/10.31234/ osf.io/y38m9 


\section{Gabriel Horn Iwaya}

https://orcid.org/0000-0002-1075-9294

Doutorando em Psicologia pela Universidade Federal de Santa Catarina (UFSC).

E-mail: gabrieliwaya@hotmail.com

\section{Janaína Gularte Cardoso}

https://orcid.org/0000-0002-2017-1304

Professora Adjunta do Curso de Administração da Universidade Federal da Fronteira Sul (UFFS); Doutoranda do Programa de Pós-Graduação em Administração da Universidade Federal de Santa Catarina; Pesquisadora do Grupo de Pesquisa/CNPq - Núcleo de Inteligência Competitiva Organizacional em Marketing e Logística (NICO/UFSC). E-mail: janaina.cardoso@uffs.edu.br

\section{João Henriques de Sousa Júnior}

iD

https://orcid.org/0000-0001-8589-8101

Doutorando em Administração pela Universidade Federal de Santa Catarina (UFSC); Mestre em Administração pela Universidade Federal de Pernambuco (UFPE); Especialista em Gestão Pública pelo Instituto Federal de Pernambuco (IFPE); Graduado em Administração pela Universidade Federal de Pernambuco (UFPE); Pesquisador do Grupo de Pesquisa/CNPq - Núcleo de Inteligência Competitiva Organizacional em Marketing e Logística (NICO). E-mail: sousajunioreu@hotmail.com

\section{Andrea Valéria Steil}

https://orcid.org/0000-0001-7853-6532

Professora Associada do Departamento de Psicologia, do Programa de Pós-Graduação em Psicologia e do Programa de Pós-Graduação em Engenharia e Gestão do Conhecimento da Universidade Federal de Santa Catarina (UFSC); Líder do Grupo de Pesquisa/CNPq - Conhecimento, Aprendizagem e Memória Organizacional (KLOM). E-mail: andrea.steil@ufsc.br 